\title{
Overlap between maculopapular exanthema and DRESS among cutaneous adverse drug reactions in a dermatology ward (2008-2012)
}

\author{
Miguel Pinto Gouveia*, Ana Gameiro, Inês Coutinho, Neide Pereira, Margarida Gonçalo \\ From 6th Drug Hypersensitivity Meeting (DHM 6) \\ Bern, Switzerland. 9-12 April 2014
}

\section{Background}

Immune-mediated cutaneous adverse drug reactions (CADR) present under different clinical patterns, some different from the main phenotypes of CADR. Our objective is to characterize manifestations and culprit drugs in CADR that required hospitalization, particularly exanthema associated with few systemic symptoms without fulfilling the European DRESS criteria (MP/DR).

\section{Methods}

A retrospective study evaluated patients hospitalized in a Dermatology ward between 2008-2012. Sex, mean age, culprit drugs, latency period, exanthema characteristics and systemic symptoms were evaluated comparing MP/DR with MPE and DRESS.

\section{Results}

We analyzed 132 patients $(85 \mathrm{~F} / 47 \mathrm{M}$; mean age of 63.98 \pm 17.68 years) with maculopapular exanthema (MPE) (21.2\%), DRESS (28.0\%), overlap MP/DR (25.8\%), Stevens-Johnson syndrome/toxic epidermal necrolysis (12.1\%), acute generalized exanthematous pustulosis (8.3\%), fixed drug eruption (3.78\%) and urticaria/angioedema $(0.76 \%)$, caused mainly by allopurinol (36.5\%), antimicrobials (30.8\%) and anticonvulsants (14.4\%). MPE occurred in 28 patients, $21 \mathrm{~F} / 7 \mathrm{M}$, mean age of $68 \pm 19,71$ years; DRESS in 37 patients, $25 \mathrm{~F} / 12 \mathrm{M}$, mean age of $61,38 \pm 18,07$ years, and MP/DR in 34 patients, $13 \mathrm{M} / 21 \mathrm{~F}$, mean age of $64.06 \pm 17.06$ years, with no significant difference between sex or age. In MP/DR, generalized exanthema was associated with face involvement in $73.5 \%$ vs $89.2 \%$ in DRESS. Liver was affected in $61.8 \%$ vs
78.4\% in DRESS, with mild cytolysis in $90.5 \%$ (median highest ALT value $109 \mathrm{IU} / \mathrm{L}$, Interquartile range (IQR) 75-162) and mild cholestasis in 61.9\% (medium highest GGT 196 IU/L, IQR 87-337), values usually inferior to DRESS (median ALT 131 (IQR 92-392) and GGT 211 (IQR 110-519). Eosinophilia occurred in $11.8 \%$ of MP/DR vs $78.4 \%$ in DRESS, with an average relative eosinophilia of $18.8 \% \pm 12 \%$ vs $16 \% \pm 7 \%$ in DRESS. Allopurinol was the most frequent culprit drug both in MP/DR (38.5\%) and DRESS (51.1\%), antibiotics were the culprit in $47.5 \%$ of MPE, $26.9 \%$ of MP/DR and $20.7 \%$ of DRESS. Latency period in MP/DR (18.06 \pm 13.17 days) was significantly longer than in MPE (11.04 \pm 9.33$)$, however shorter than DRESS $(23.85 \pm 13,80)$.

\section{Discussion and conclusion}

We identified a MP/DR overlap syndrome with many features suggesting a less severe DRESS, a mini-DRESS, in a continuum spectrum among exanthematous CADR, with MPE and DRESS as the two poles of the spectrum.

Published: 18 July 2014

doi:10.1186/2045-7022-4-S3-O6

Cite this article as: Gouveia et al:: Overlap between maculopapular exanthema and DRESS among cutaneous adverse drug reactions in a dermatology ward (2008-2012). Clinical and Translational Allergy 2014 4(Suppl 3):06. 